\title{
Conception and State of the Radiometric Analysis Breadboard (RAB) for the Mercury Radiometer and Thermal Infrared Spectrometer (MERTIS)
}

\author{
T.Saeuberlich, E.Lorenz, W. Skrbek \\ German Aerospace Center, Optical Information Systems, Rutherfordstr. 2, 12489 Berlin,Germany
}

\begin{abstract}
As a part of the ESA deep space mission to mercury - BepiColombo - investigations of mercury's surface layer using a push-broom thermal infrared imaging spectrometer (MERTIS) with a high spectral resolution is planned. One of the scientific goals is the measurement of Christiansen Features which are emissivity maxima resulting from rapid changes in the real part of the mineral's refractive index. Their positions within the spectral range of 7-14 $\mu$ m deliver information about mineralogical compositions. For these measurement MERTIS needs to have a high spectral resolution of 90nm. The planet will be mapped with a resolution of $500 \mathrm{~m}$ and a $\mathrm{S} / \mathrm{N}$ ratio of at least 100 . For the measurement of the surface radiation a micro-bolometer detector array will be used. A detectivity of 1.0E9 is required. High sensitive TIR systems commonly use cooled detectors with a large mass budget and high electrical power consumption. One of the challenges of MERTIS is the use of an uncooled micro-bolometer detector. The development of MERTIS is currently in an early phase but a breadboard concept will be presented. Special attention is payed to the first of two phases of the breadboard concept:
\end{abstract}

- The Radiometric Breadboard (RAB) has been configured for the development of the opto-electronical components and for the investigation of radiometric calibration methods and algorithms. The design of the RAB is already a spectrometer configuration but it cannot reach the performance the technical and scientific requirements demand.

- The Spectro-Radiometric Breadboard (SRB) will be implemented for investigations of the performances of the optics and detector of MERTIS. Relevant components have to be developed and validated particularly in the spectral domain. The SRB will be the prototype of MERTIS.

Keywords: MERTIS, Breadboard, Imaging spectrometer, Bolometer array, Infrared spectrometer, Push-Broom

\section{INTRODUCTION}

One part of ESA's BepiColombo deep space mission to Mercury will be the Mercury Radiometer and Thermal Infrared Spectrometer (MERTIS) developed by the German Aerospace Center (DLR) in Berlin-Adlershof. MERTIS is an IRimaging spectrometer based on the pushbroom-principle [1] having the following scientific objectives [2]:

Study of Mercury's surface composition

Identification of rock-forming minerals

Mapping of the surface mineralogy

Study of surface temperature and thermal inertia

The knowledge of the surface of Mercury is based on the Mariner 10 data (50\% surface coverage) and ground based observations [2]. Up to now only very few spectra of Mercury are available which result from earth-based telescopic measurements [3]. The spectra have a very limited spatial resolution and are often affected by observational problems resulting from the planet's proximity to the Sun and/or from telluric absorption. It is the goal of this experiment to provide detailed information about the mineralogical composition of Mercury's surface layer by measuring the spectral emittance of different locations in a broad spectral range.

Mercury's thermal emission at day side starts to dominate the all over radiance already at wavelength $>1.2 \mu \mathrm{m}$ (at $725 \mathrm{~K}$ ) depending on the surface albedo. The range between 0.8 and $2.8 \mu \mathrm{m}$ is a transition region characterized by the overlapping of the reflected solar radiation and the thermal emission. Because Mercury's thermal flux is higher than the flux reflected from its surface, near infrared spectroscopy is very difficult but emittance spectroscopy in the thermal IR 
range $7-14 \mu \mathrm{m}$ becomes possible. This range has a high potential for the identification of the major rock-forming minerals because they have their fundamental vibration bands within there. More detailed information can be obtained from [4]. While globally mapping the planet with a spatial resolution of 500m and a S/N ratio of at least 100, MERTIS will investigate this spectral range with a resolution of ca. $90 \mathrm{~nm}$ in order to be able to identify the mentioned features.

Because of the hot environment of Mercury and the spectral range of 7-14 $\mu \mathrm{m}$ and to save mass and power consumption an uncooled Micro-Bolometer detector array will be used. These detectors are available from the US and in Europe up to a size of standard chips of 640 x 480 pixels and pixel sizes of $50 \mu \mathrm{m}$ presently and down to $25 \mu \mathrm{m}$. Using an uncooled Micro-Bolometer will be one of the MERTIS challenges.

The main performance requirements for the MERTIS instrument derived from the scientific requirements are summarized in the following table (for more detail see [4]):

\begin{tabular}{|l|l|}
\hline Spectral coverage & $7-14 \mu \mathrm{m}$ \\
\hline Spectral resolution & $<90 \mathrm{~nm}$ \\
\hline S/N for spectral range $7-14 \mu \mathrm{m}$ & $>100$ \\
\hline Spatial resolution for global mapping & $500 \mathrm{~m}$ \\
\hline Targeted observation with better than $500 \mathrm{~m}$ & $5-10 \%$ of the surface \\
\hline
\end{tabular}

Table 1: MERTIS performace requirements

\section{METHODICAL INVESTIGATIONS}

There are several possible classes of imaging spectrometers each having different methods for the extraction of the spatial and spectral information [1]. MERTIS will use the pushbroom-principle where a 1D-FOV is imaged to the whole detector array and where a line corresponds to the spatial information and a column to the spectral information of the object scanned. The spectral splitting will be done using a reflective diffraction grating. The 1D-FOV lies perpendicular to the orbiter track and each frame will be recorded after the movement of the orbiter over a certain spatial distance or time (along-track scanning).

In this way MERTIS's gathers a 3D-information for each scanning step including spatial, spectral and radiometric properties of the observed object. The information determined must match the requirements mentioned in Table 1 in order to fulfill the scientific requirements. Especially the optimization of the SNR to reach a value higher than 100 in the spectral range $7-14 \mu \mathrm{m}$ with a spectral resolution of $90 \mathrm{~nm}$ will be a challenge. Therefore the spectrometer concept needs in the early breadboarding phase investigations regarding the following topics:

\section{Radiometric Characterization:}

The instrument output signal is the sum of the following parts of radiation power:

- $\quad$ input radiation power to be analysed and which is proportional to the $\mathrm{f}$ of the instruments optical path and the scene temperature $(\mathbf{C 1})$

- $\quad$ detector housing radiation power proportional to the detector field of view covered by the radiation power housing within the detector dewar (C2) and depending on the housings emissivity and temperature

- $\quad$ stray light due to the instruments temperature (C3)

$D N_{\text {OUT }}=C 1 * \int_{\lambda k, \Delta \lambda} L\left(T_{I N}\right) d \lambda+C 2 * \int_{\lambda 1}^{\lambda 2} R(\lambda)_{D E T} L\left(T_{D E T H O U S I N d}\right) d \lambda+C 3 * \int_{\lambda 1}^{\lambda 2} R(\lambda)_{D E T} L\left(T_{I N S T R}\right) d \lambda$

or for each pixel $(\mathrm{k}, \mathrm{l})$ :

$$
D N_{\text {OUT, }, l}=c_{k, l} * L\left(T_{I N}\right)+D N_{\text {OFFSET }}\left(T_{\text {DETHOUSING }} T_{\text {INSTR }}\right)_{k, l}
$$

Measurements with a bolometer camera in a thermal chamber have verified that there is in fact a linear dependence between the offset and the measured signal DN. In contradiction to the sensitivity (c1) the offset $\left(C 2 * \int \ldots+C 3 * \int \ldots\right)$ 
shows a strong dependence to the temperature state of the instrument. It is the goal for the calibration of the instrument to determine the sensitivity $\mathrm{c} 1$ and (c2,c3) which are according to the linear model (1) constants characterizing the effect of the (temperature depending) instrument radiation on the measured signals.

The determination of the radiometric coefficients for each pixel is done with a simple two point calibration:

$c_{k, l}=\frac{D N(T 1)_{k, l}-D N(T 2)_{k, l}}{L\left(T 1, \lambda_{k}\right)-L\left(T 2, \lambda_{k}\right)}$

The offset can be measured using a blackbody with a known temperature located at the input port of the spectrometer:

$$
\operatorname{DN}\left(T_{\text {INST }}\right)_{\text {OFFEET, } k, l}=D N_{\text {OUT }, k, l}-c_{k, l} * L\left(T_{B B}\right)
$$

From (2) the input radiation can be calculated if we have an updated measurement of the offset. This has to be ensured prior or after each measurement:

$$
L_{k, l}\left(T_{M E A S}\right)=\frac{D N_{O U T, k, l}-D N_{O F F S E T, k, l}}{c_{k, l}}
$$

$\begin{array}{ll}L & \text { spectral radiance by Planck-Function } \\ \lambda & \text { wavelength } \\ C & \text { calibration coefficients } \\ T & \text { blackbody temperature } \\ D N & \text { output signal of the instrument }\end{array}$

\section{Spectral assignment:}

The goal of the spectral assignment is the determination of the wavelength assignment to the spectral line $\mathrm{k}$ of the detector array. A parallelized light beam of a monochromator must illuminate the input port of the instrument. The local maxima over the matrix lines $(\mathrm{k})$ have to be determined for different wavelengths. A linear fit of the different center wavelengths $\lambda$ gives coefficients (a,b) of the assignment function:

$$
\lambda(k)=a k+b
$$

\section{Spectral resolution:}

The spectral resolution is defined as the measured spectral response with the parameter Full Width at Half Maximum (FWHM) of the Bessel fitting curve. The common problem to find a narrow band source with a sufficiently small bandwidth for the thermal infrared has to be solved.

\section{Geometric calibration:}

The main task of the geometric calibration is the exact determination of the sensor's pixels line of sight orientations (LOSO) relative to the principal axis. The pixel LOSO is determined by measuring of the two angles $(\gamma, \delta)$ for line k of the nodal bench during illumination of a certain number of pixels. Fitting the maxima of these measurements to a second or third order function gives all pixel's LOSOs. A second part of the geometric calibration is the determination of the angle divergence of the alignment mirrors to a reference pixel. The alignment mirrors will be used for the determination of the orientation of the line of sight of the different instruments on the spacecraft. 


\section{Geometric resolution:}

The spatial resolution is a spatial width defined as the minimal resolvable distance between two points. This measurement is depending on the quality of the optical path and the dimensions of detector elements. The measured Point-Spread-Function (PSF) can deliver the necessary parameters. It is convenient to define the resolvability of a two point object as the 50\% intensity level of the PSF (full width at half maximum FWHM).

\section{BREADBOARDS \\ 3.1 CONCEPT}

The required instrument performance must be verified and optimized in order to achieve the scientific goals. Test and calibration procedures have to be implemented and performed during the different instrument development phases. The procedures have to deliver a complete knowledge of the instrument behaviour and produce a relevant set of reference data for the interpretation of the results during the mission. The calibration procedures to be achieved make an intense breadboarding phase necessary. The MERTIS breadboarding concept is therefore divided into two phases:

- The Radiometric Breadboard (RAB) has been configured for the development of the opto-electronical components and for the investigation of radiometric calibration methods and algorithms. The design of the $\mathrm{RAB}$ is already a spectrometer configuration, but it cannot reach the performance the technical and scientific requirements demand.

- The Spectro-Radiometric Breadboard (SRB) will be implemented for investigations of the performances of the optics and detector of MERTIS. Relevant components have to be developed and validated particularly in the spectral domain. The SRB will be the prototype of MERTIS having all of its design features. More details about the MERTIS design can be found in [6].

\subsection{RADIOMETRIC ANALYSIS BREADBOARD 3.2.1 CONFIGURATION}

The RAB is the currently realized breadboard. Its key component is a F\# 7 monochromator which has been modified to a spectrometer for the radiometric measurements. The main task is the radiometric analysis under spectral conditions. With this configuration radiometric parameters have to be measured by means of a spectral known source (e.g. spectral

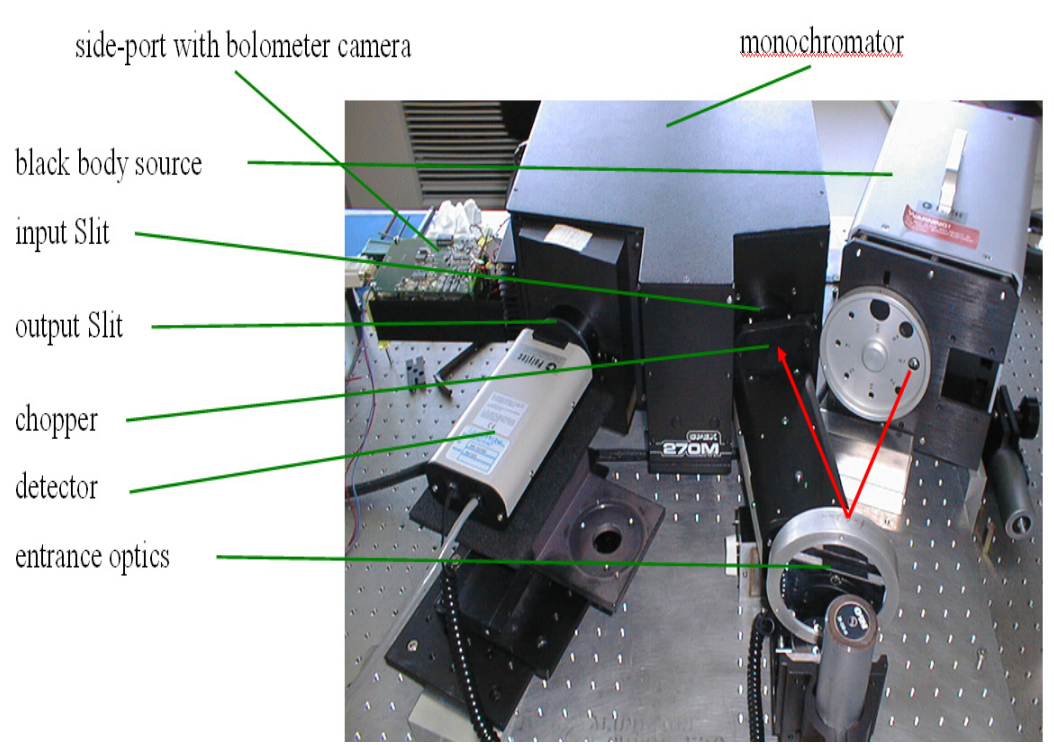

Figure 2a: RAB configuration coefficients of the sensitivity of the array pixel).

Fig.2a shows an arrangement of RAB consisting of:

- $\quad$ Black Body Source (optional with shutter)

- Entrance Optics

- $\quad$ Monochromator with in- and output slit

- $\quad$ TIR-Camera Module OIS-IR3 at side port

- $\quad$ Single pyroelectric detector at output slit

Fig.2b shows additionally the inner parts of the monochromator:

- $\quad$ Shutter

- Monochromator Optics 


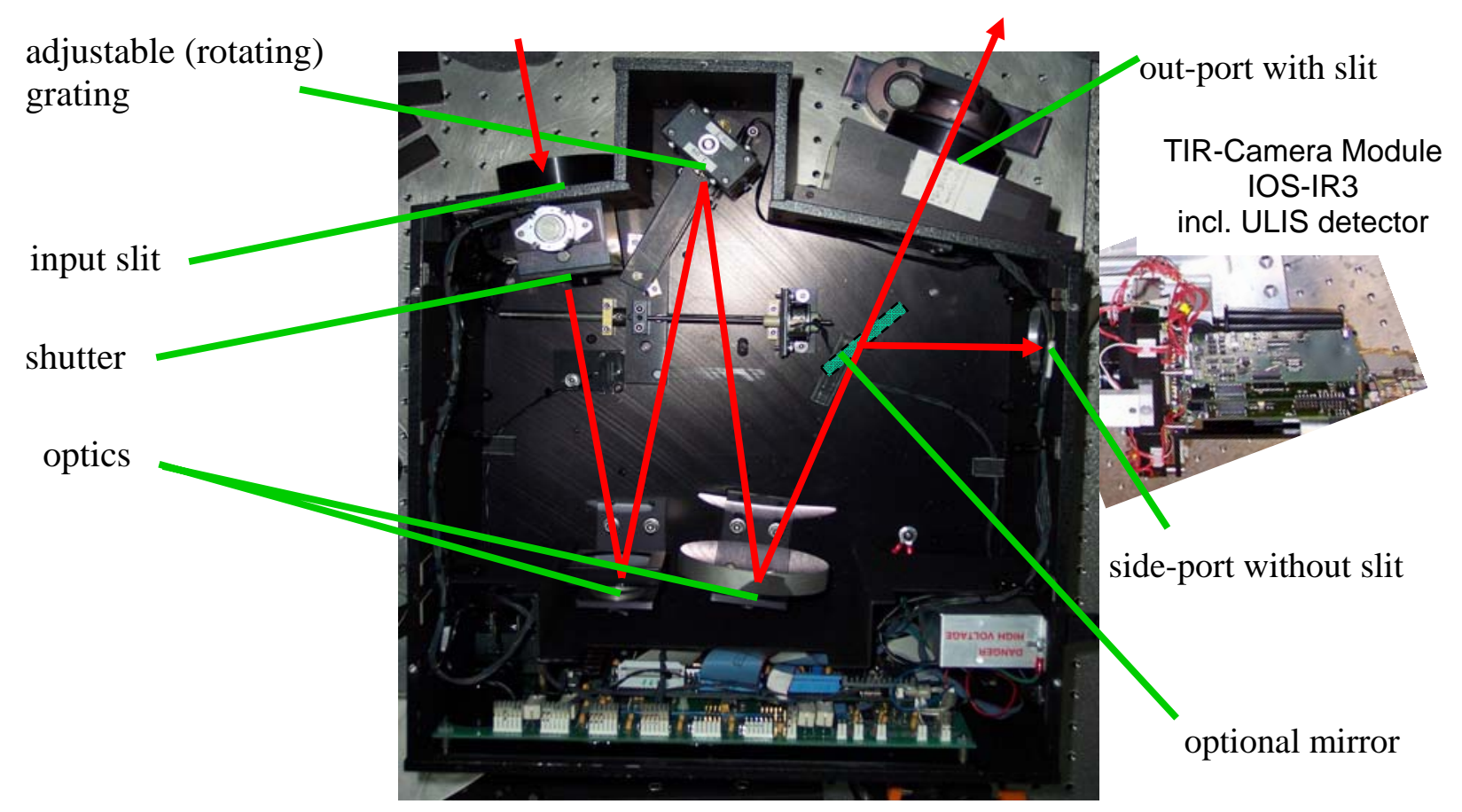

Figure 2b: RAB- Spectrometer - view inside the monochromator

Base line of the RAB is a blazed diffraction grating which is a plane glass substrate coated with aluminium. It has ruled grooves with a density of 14.3 lines $/ \mathrm{mm}$ on an area of $46 \times 46 \mathrm{~mm}^{2}$ and a blaze angle of $3.3^{\circ}(=>$ blaze wavelength at $8.2 \mu \mathrm{m})$. The grating is one of the key components of the system. It has a strong effect on the spectral system response function as it will be discussed in 3.2.4. The modeling of the spectral path (Fig.2c) shows that the dispersion of the available standard grating (60 lines $/ \mathrm{mm}$ ) is too large. With the modeling of this grating its possible to obtain sequentially spectra of $4 \mu \mathrm{m}$ band width within a range of 7 to $15 \mu \mathrm{m}$ for center wavelengths controlled by the EGSE. The spectral resolution has to be verified with a special narrow band source.

The detector of the OIS-IR3 is a bolometer array of $320 \times 240$ elements with 50 $\mathrm{m}$ pitch and a Noise Equivalent Temperature Difference (F\#1-NETD) of $0.1 \mathrm{~K}$. A Critical part is the thermo stabilizing of the detector by a Thermo Electrical Cooler (TEC). To achieve the

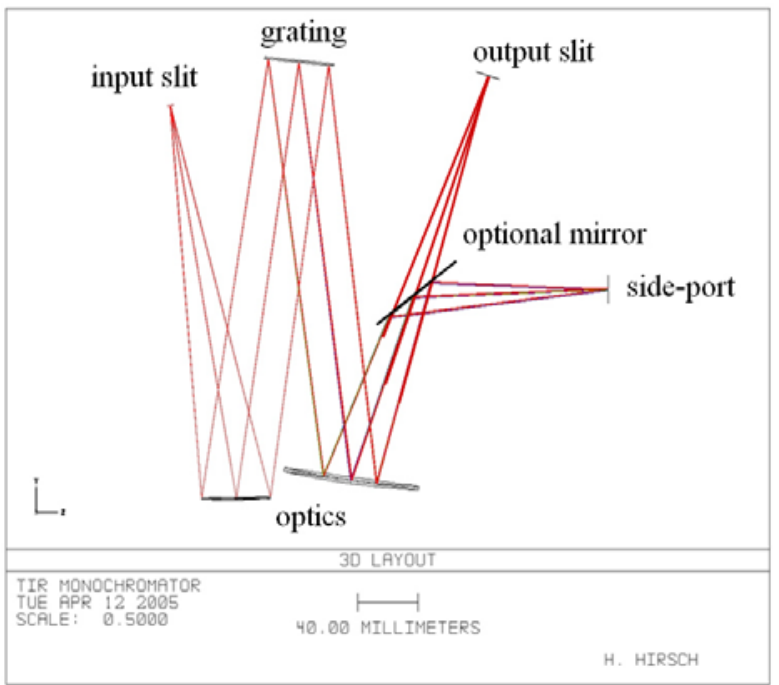

Figure 2c: Optical Layout of RAB required $10 \mathrm{mK}$ stability a special TEC algorithm at the camera controller had been developed and implemented for the MERTIS breadboards (s.3.2.3).

\subsubsection{WAVELENGTH ASSIGNMENT}

The adjustable (by changing the tilt angle) grating used in the RAB is driven by a stepper motor. To do spectrally resolved measurements a wavelength assignment is required. This procedure determines the assignment of the stepper motor position to: 
a) the center wavelength at the monochromator output slit

b) a wavelength corresponding to a pixel row of the bolometer detector array at the monochromator side port

\section{a) Output slit assignment:}

For this procedure a choppered single pyroelectric detector from a commercial distributor was placed directly in front of the outputslit of the monochromator and the chopper device in front of the input slit.

Because of the reflective optical components of the RAB-arrangement the slit output calibration can be performed by a narrow band calibration source which works in the visible (VIS) spectral range. The following procedure is based on the properties of the grating equation:

$$
m * \lambda=d^{*}(\sin \alpha+\sin \beta)
$$

where $\lambda$ is the wavelength observed in the m-th order of diffraction for a given set $(\alpha, \beta)$ of angles of incidence and reflection (i.e. observation). $\mathrm{d}$ is the width of the groove structure which is a constant. For a fixed angle of incidence $\alpha$ (which is the case for a certain stepper motor position) and a certain wavelength $\lambda$ we observe $\lambda$ in each order of diffraction at different angular positions $\beta_{\lambda m}$. For each set $(\alpha, \beta)$ many different sets (m, $\lambda$ ) can fulfill the grating equation. This is because of the different orders of diffraction overlap each other. $\lambda$ in the first order is located at the same angular position as $\lambda / 2$ in the second order, $\lambda / 3$ in the third and so on. Vice versa - the angular position of $\lambda$ in the $\mathrm{m}$-th order is the same as the angular position of a wavelength $\lambda * \mathrm{~m}$ in the first order. The first order is the interesting one, because the grating used in the RAB is optimized for it.

This principle can be used to do a wavelength calibration using a monochromatic light source. For the RAB wavelength calibration a stabilized laser source of $633,429 \mathrm{~nm}$ has been used. The monochromatic incident light is spread by the grating into the different orders of diffraction (observer can see spots) covering a certain angular position interval. To assign these spots to the corresponding order of diffraction $m$ it is necessary to identify the 0-order (easy to find because in the 0 -order the grating is acting like a mirror) and then just counting the neighbouring spots.

Leading each of the spots (indexed with $\mathrm{m}$ ) to the output slit (by tilting the grating i.e. driving the stepper motor) delivers an assignment of the motor position to a wavelength $\lambda * \mathrm{~m}$. Fig. 3 shows the signal at the output slit over the step number of the grating adjustment. Selecting the peaks and the corresponding wave length we get a wave length calibration equation for the output slit port by a linear fit for these peaks:

Wavelengths/ $\mu \mathrm{m}=2,6432$ step $-4951,42$

Figure 3: Measured intensity over stepper motor position. Each peak corresponds to the $633,429 \mathrm{~nm}$ HENE-LASER line in a certain order of diffraction $\mathrm{n}$. Using the properties of the grating equation it is possible to do an assignment of the wavelength n*633,420nm to a stepper motor position.

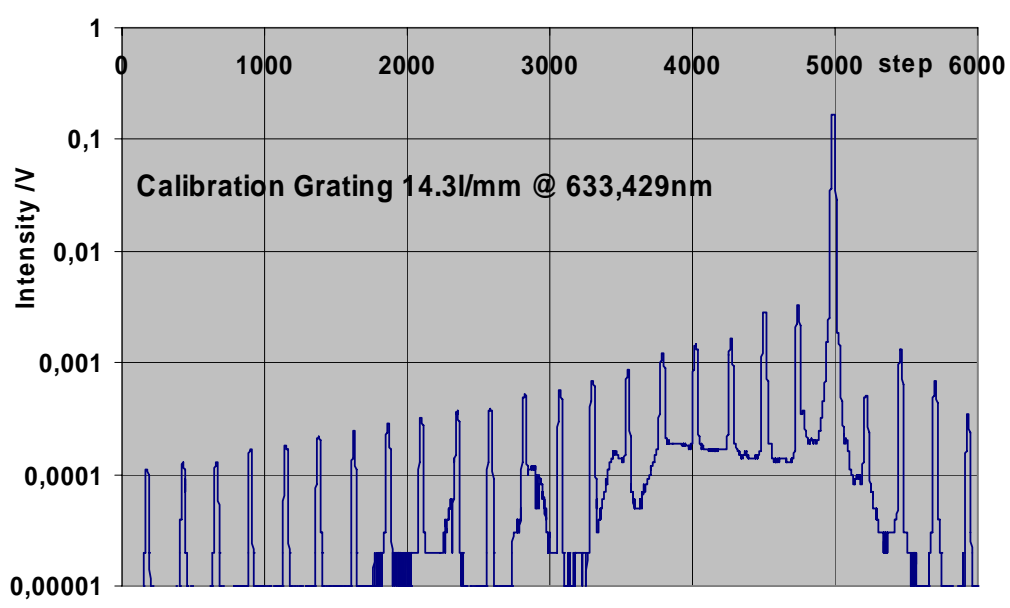


Another important task is to determine the 0-position of the stepper motor. The monochromator uses for that purpose a mechanical switch which produces an uncertainty of $0.3 \mu \mathrm{m}$. To reduce that error or not at the RAB-level is still under discussion. The slit output procedure delivered an uncertainty of $12.5 \mathrm{~nm}$.

\section{b) Detector array assignment:}

After the wave assignment of the output slit port the output mirror has been located in the monochromator in front of the side (lateral) port deflecting the light beam to the bolometer array (Fig.4).

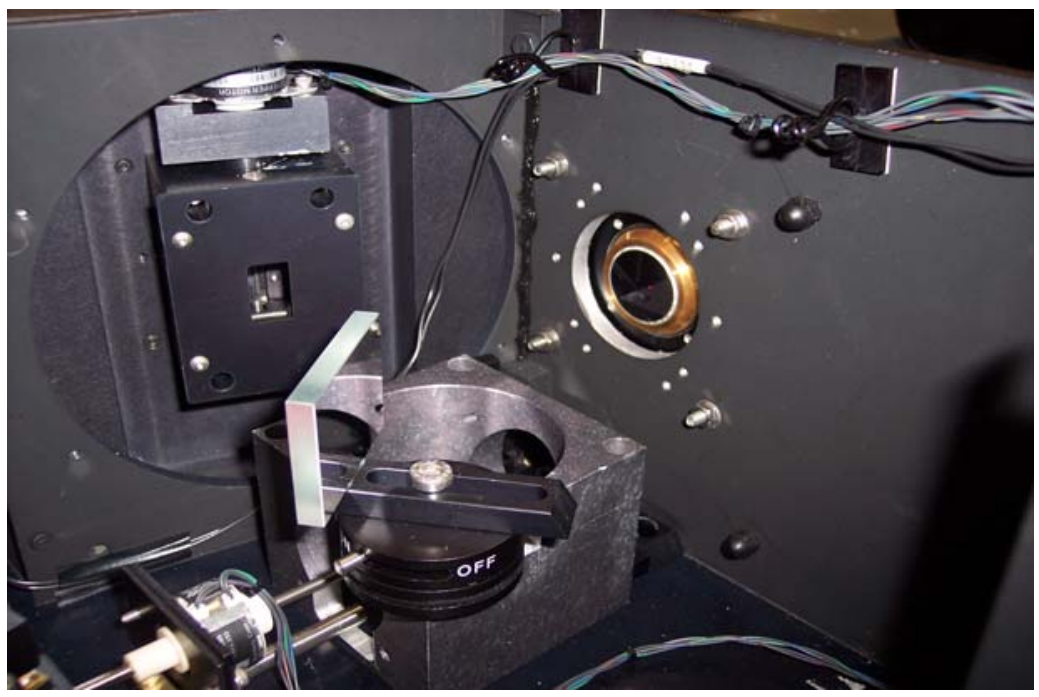

Figure 4: Side port with output mirror and bolometer

Fig. 5 shows the light spot sequence of the higher order spots at the location of the focal plate. These spots cannot be detected by the IR detector but this measurement can be used to determine the rough position of the spectra in the focal plane.

To identify the zero position the VIS laser will be replaced by the IR source (Black Body source). The zero position is the zero order IR-image of the input slit (Fig.6). This image gives the possibility to adjust both the wavelength and the sharpness of the RAB spectrometer in the spectral and in the spatial domain.

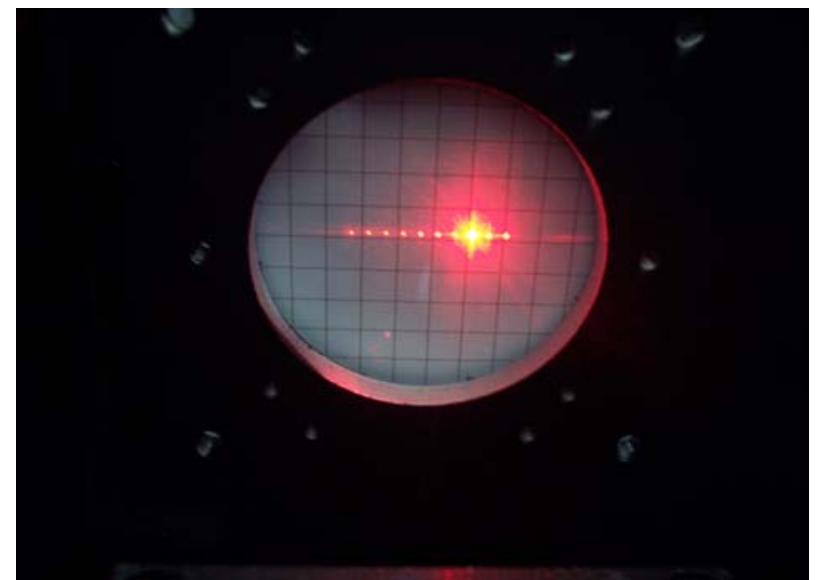

Figure 5: Sequence of higher order spots at the location of the focal plate

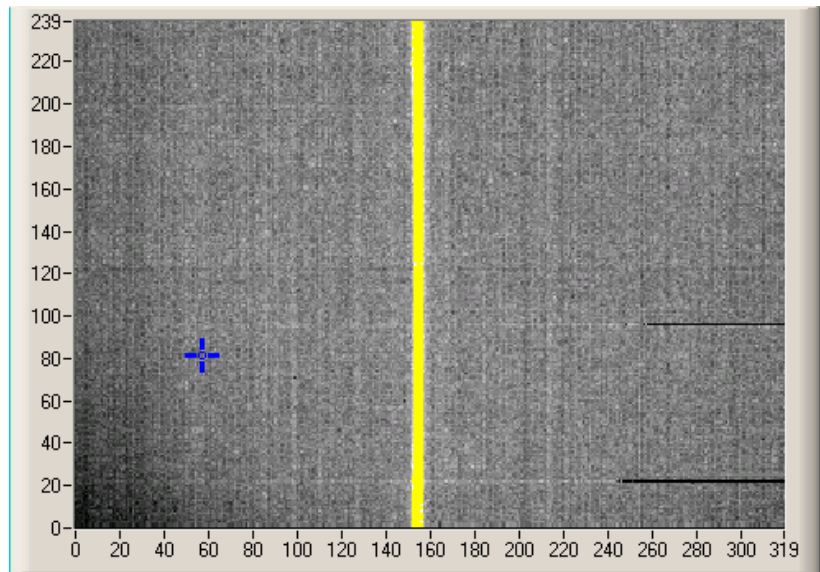

Figure 6: Image of the input slit $0.1 \mu \mathrm{m}$ at $9 \mu \mathrm{m}$ 


\subsubsection{THERMAL STABILIZATION OF THE DETECTOR CHIP}

The sensitive elements of the bolometer array are coupled to the read out circuit which works as large multiplexer structure. All these elements have their own offset and their individual sensitivity. These parameters depend on the chip temperature. To get stable calibration functions or tables the bolometer chip temperature has to be stabilized. The manufacturer recommends $10 \mathrm{mK}$ in the region of $30^{\circ} \mathrm{C}$. A special algorithm has been developed and implemented in the FPGA of the OIS camera to control the chip temperature by the Peltier Thermo-Electrical Controller (TEC) of the Bolometer chip. The first tests show a good stability for room temperatures in the required order [fig.7]. The special algorithm has been adapted to the non linear behaviour of the NTC chip temperature sensor. Because of the large noise of this sensor it was necessary to implement a Kalman filter for noise reduction.

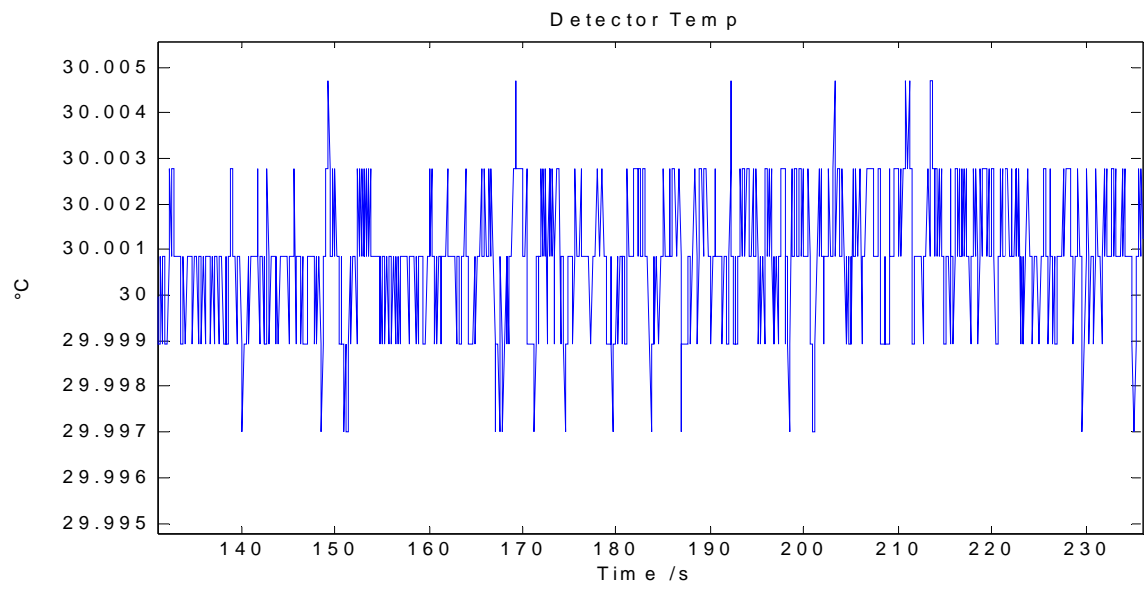

Figure 7: Chip temperature stabilisation

Environmental temperature and long term tests have to be performed to qualify this stabilization algorithm.

\subsubsection{MEASUREMENT OF THE SPECTRAL RESPONSE WITH A SINGLE PYROELECTRIC DETECTOR}

One of the important goals is the spectro-radiometric validation of the RAB with the grating as key component. The spectral response of the optical components determines the spectro-radiometric behaviour of the RAB. The spectral system response is mainly determined by the grating efficiency which can be modeled using Fraunhofer Diffraction Theory [7]. To go the first step of calibration it is useful to validate RAB with a commercial choppered radiometer (Fig.2a).

The spectral response $R(\lambda)$ can be calculated out of the detector signal measured S and BB temperature T which goes together with $\lambda$ into the calculation of the radiant emittance $W$ for blackbodies according to Planck's Law:

$\mathrm{S}=R(\lambda) * W(\lambda, T)$

For the first measurement the input port of RAB was stimulated with the Black Body (BB) source SR20 in the range of 300 to $1000^{\circ} \mathrm{C}$. No higher order filters were used. An assumption was that the offset of these measurements was compensated completely by the choppered radiometer. But Fig.8a shows that there are still large differences of the responses between the different $\mathrm{BB}$. The discussion of this result led to the idea to consider a wavelength dependent offset $W_{0}(\lambda)$. With the data from two measurements at different temperatures $\left(T_{1}, T_{2}\right)$ we get a linear set of two equations and can calculate the spectral response $R(\lambda)$ and the spectral offset $W_{0}(\lambda)$ :

$S_{1}=R(\lambda) *\left(W\left(\lambda, T_{1}\right)-W_{0}(\lambda)\right)$

$S_{2}=R(\lambda) *\left(W\left(\lambda, T_{2}\right)-W_{0}(\lambda)\right)$ 


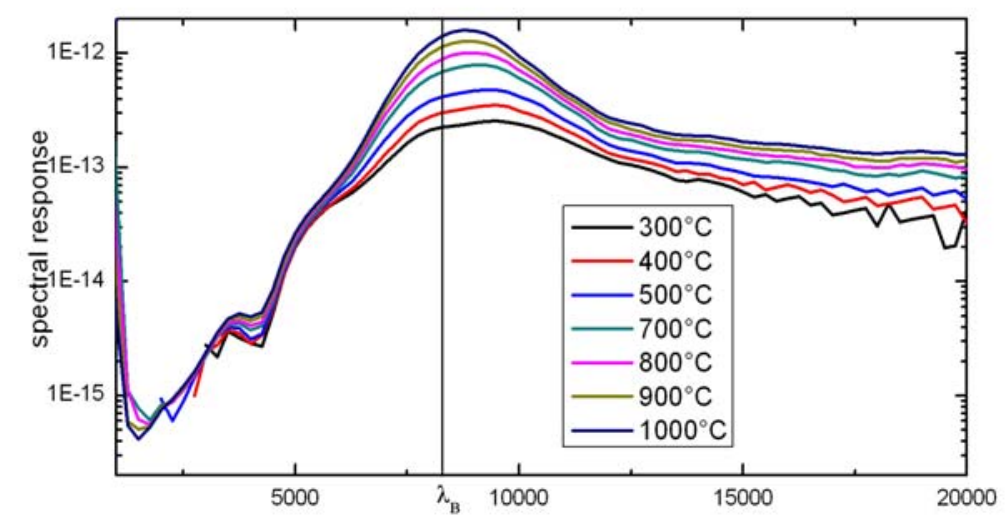

Figure 8a: Response calculated with constant offset

(shape error 7.8\%)

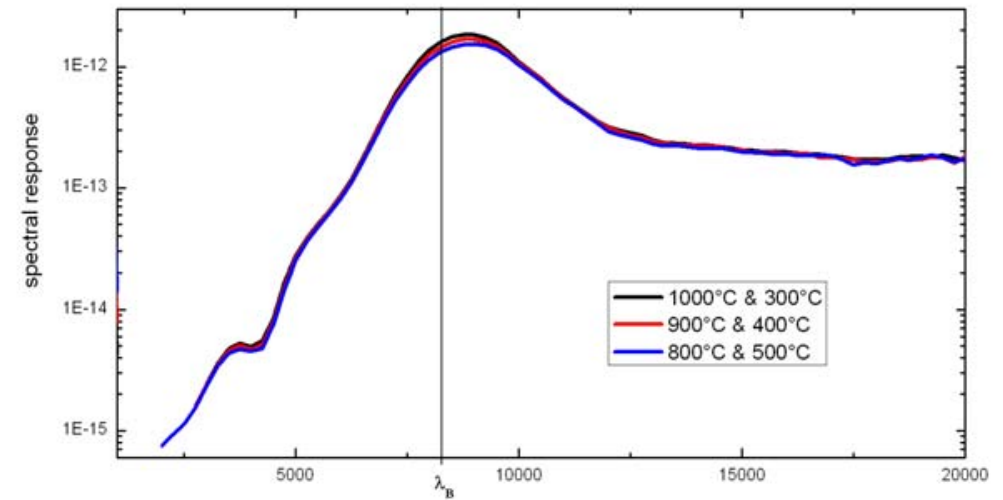

Figure 8b: Response considering wave length dependent offset (shape error 3.73\%)

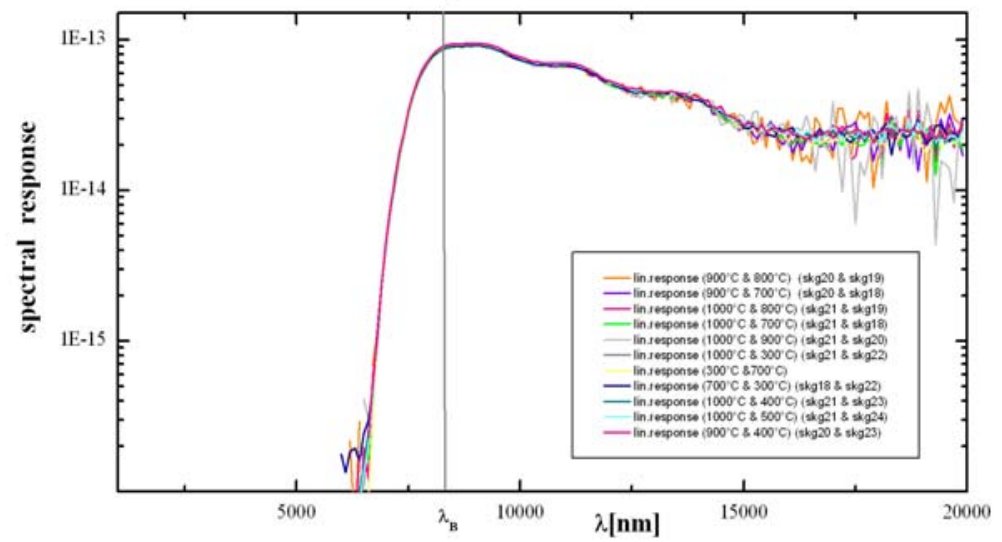

Figure 8c: Response considering wave length dependent offset and using long-pass IR filters with $7.3 \mu \mathrm{m}$ cut-on wave length (shape error $0.42 \%$ )

The result of this calculation - shown in Fig.8b. - is obviously better. The quantitative progress can be evaluated by a shape error factor which goes from 7.8\% (Fig.8a) down to 3.73\% (Fig.8b). This improvement results from offset variations caused by different wave length positions of the grating. The calculations done for measurements without higher order filter could now be applied to measurements using a long-pass filter with a cut-on wave length of 7.3 $\mu \mathrm{m}$. The filter was installed between the output slit and the detector. The resulting responses (Fig.8c) show the improvement. The shape error factor went down to $0.42 \%$ and clarifies the effect coming from the higher order radiation.

The calculated response also shows that the grating caused a strong descent of radiation power above $12 \mu \mathrm{m}$. This behaviour can be approximately confirmed by the mentioned Fraunhofer Diffraction Theory [7]. 


\subsubsection{MEASUREMENT OF THE SPECTRAL RESPONSE WITH THE BOLOMETER ARRAY}

The procedure for the determination of the spectral response function using a single pyroelectric detector was modified and applied to whole bolometer detector array. For this measurement the grating stayed in a fixed position where the middle column (pixel column 160 of the $320 x 240$ pixel array) corresponds to $9.12 \mu \mathrm{m}$. The spectral system response functions calculated from the measurements with the single pyroelectric detector (calibrated by vendor) describe the spectro-radiometric behaviour of the whole system without detector. For the bolometer measurement Fig. 9 shows exemplary the resulting frame recorded at a $\mathrm{BB}$ temperature of $1200^{\circ} \mathrm{C}$. For this measurement 200 frames were taken and their

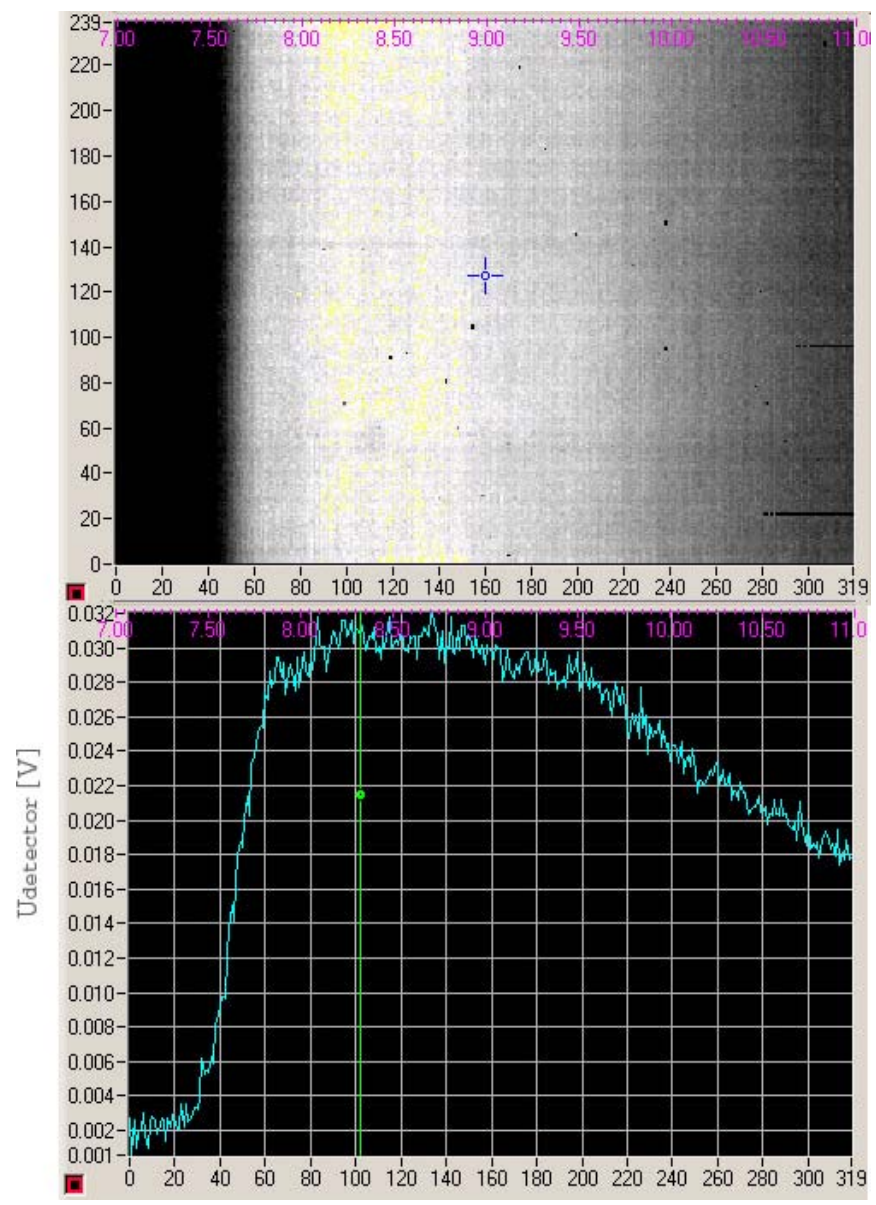

Figure 9: upper picture: mean frame recorded using the bolometer detector array. Grating was driven to $9.12 \mu \mathrm{m}$ @ pixel column 160, BB @ $1200^{\circ} \mathrm{C}$; lower picture: measured voltages of each pixel of line 100 $12.5 \mathrm{~nm}$. The calculation which was applied for the single detector system can be done for each pixel column of the bolometer detector array. The window in front of the detector chip serves as a long-pass filter with ca. 7.5 $\mu \mathrm{m}$ cut-on. To calculate the response all measuring values for each pixel from one column (which corresponds to the same wavelength

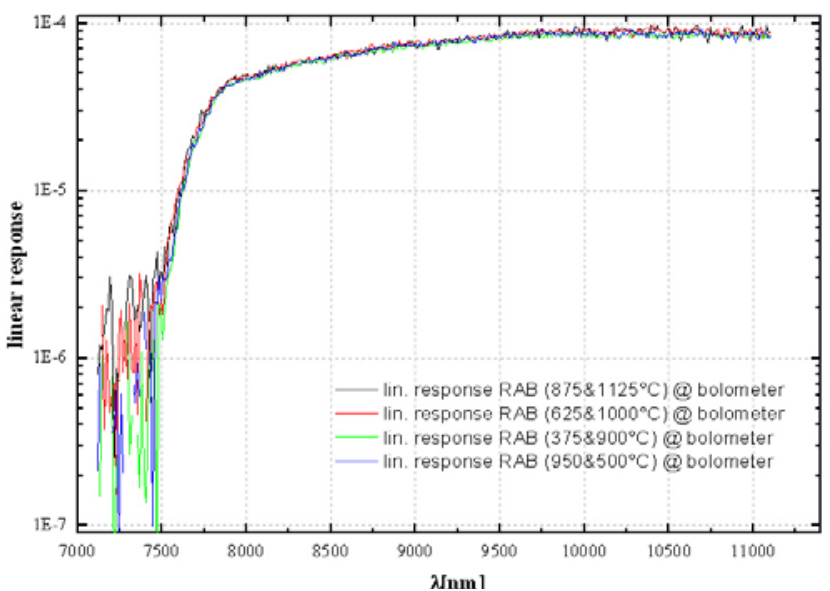

Figure 10: Spectral response functions calculated out of bolometer measurements with different BB temperatures (shape error 3.41\%)

mean value calculated to reduce the temporal noise. The same procedure was done with a closed input shutter which produced a dark mean-frame. In fig 9. the upper picture shows the difference between both measurement and darkmeasurement (additive pattern correction). For the next investigations the individual pixel sensitivities will be considered as well (multiplicative pattern correction). The picture below shows the measured voltages of each pixel from line 100 of the detector array. Fig. 10 shows the spectral system response for the bolometer detector measurement. The reasons for the difference in the spectral behaviour can be probably the following:

For the bolometer measurement this calibration still has to be done for each pixel representing a different wavelength and having an individual sensitivity. The spectral response function for the bolometer measurement is still including the individual properties of the detector itself. Additionally - in contrast to the single detector arrangement - each pixel has a different position relative to the optics so that the geometrical properties of the optics have an influence too. Because this measurement was done with a fixed grating position, effects resulting from different angles of incidence relative to the grating do not go into the calculated spectral system response function while the spectral response functions for the single-detector arrangement contain these effects.

Disregarding the broadening depending on the width of the input slit and the spectral dispersion of the grating two pixel columns would represent a spectral sampling distance of ca. 
interval) were accumulated to one value and were used as measured signal value $\mathrm{S}$ within the same calculations as for the single detector (equ. 10). The results for the spectral response function are shown in fig 10. The shape error for the bolometer measurement has a value of $3.41 \%$ and is much worse than the results for the pyroelectric detector. Further investigations will be done to make the comparison between the spectral response functions of the pyroelectrical detector measurements and the bolometer measurements possible. For this the mentioned effects have to be considered.

\section{CONCLUSION}

The first of the two breadboards for the MERTIS development (RAB) has been configured. The adaptation of the components of the RAB has been completed. EGSE have been written for the control of the components and the data acquisition. The RAB can now be operated in a single detector and a bolometer array detector configuration. First spectral and radiometric calibration procedures and algorithms have been tested and will be optimized during the next measurements.

The linear performance of the detector depends on its temperature. Therefore a TEC-regulation algorithm was developed so that any temperature within an interval of $20-40^{\circ} \mathrm{C}$ can be set and stabilized. The regulation produces an error of ca. $10 \mathrm{mK}$ at a chip temperature of $30^{\circ} \mathrm{C}$ which is the recommended operating temperature.

A spectral assignment was done using a Visible-LASER source at the output slit of the monochromator. In this configuration the spectral resolution of the RAB is ca. $12.5 \mathrm{~nm}$. A method for a spectral assignment of the rows of a bolometer array was tested too - but it needs further investigations to achieve higher precision and to get stable results. (Spectro-) Radiometric measurements include the determination of the spectral system response function in a single detector and bolometer arrangement. The error of the response function can be evaluated by introducing a „shape error“ measure which has currently been reduced to a value of $0.42 \%$ for the single detector measurements. This improvement (shape-error was at $7.8 \%$ before) was achieved by introducing a spectral offset coefficient and using IR-long-pass filters. Identifying the effects producing the remaining shape-error and getting lower shape-error values is subject of further investigations.

\section{ABBREVIATIONS}

EGSE Electrical Ground Support Equipment

FWHW full width at half maximum

LOSO pixels line of sight orientations

MSI Multi Spectral Imager

NEdL Noise Equivalent Differential Radiance

PSF Point Spread Function

OGSE Optical Ground Support Equipment

RAB Radiometric Analysis Breadboard

SRB Spectro-Radiometric Breadboard

TEC Thermo Electric Cooler

\section{REFERENCES}

1. R.Glenn Sellar, Glenn D. Boreman, „Classification of imaging spectrometers for remote sensing applications“, Optical Enginieering 44(1),013602, January 2005

2. J.Helbert, E.Jessberger, J.Benkhoff, G.Arnold, et al., „MERTIS - A thermal infrared imaging spectrometer for the Bepi-Colombo Mission“,Lunar and Planetary Science XXXVI (2005)

3. Sprague,A.L.,Roush, T.L. (1998), Icarus, vol. 133, p. 174-183

4. "Scientific and Technical Plan Proposal MERTIS-TIS", University of Münster/DLR, Ref:MER-IFP-PP-001, 2004

5. E.Lorenz,W. Skrbek, “Calibration of a Bi-Spectral Infrared Push Broom Imager”, Infrared Space Borne RemoteSensing IX, San Diego August 2001, Proceedings SPIE Vol. 4486 pp.90-103

6. I. Walter, H. Hirsch, J. Knollenberg, H. Jahn, T.Säuberlich, H. Venus, W. Skrbek (2006): "MERTIS - the design of a highly integrated IR imaging spectrometer” EGU, General Assembly, Vienna, 2006-04-02 - 2006-04-07

7. E.S.Barrekette,R.L.Christensen, „On Plane Blazed Gratings“, IBM Journal, March 1965 